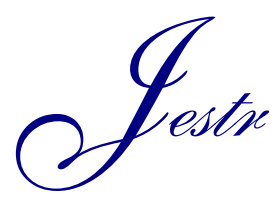

Journal of Engineering Science and Technology Review 10 (4) (2017) 109-114

Research Article

www.jestr.org

\title{
SEMG Based Classification Using Wavelet Function for Around Shoulder Muscles
}

\author{
Amanpreet Kaur ${ }^{1, *}$ and Amod Kumar ${ }^{2}$ \\ ${ }^{1}$ Thapar University, Patiala, India \\ ${ }^{2}$ CSIR-Central Scientific Instruments Organisation, Chandigarh
}

Received 12 April 2017; Accepted 29 August 2017

\begin{abstract}
The wavelet transform is the accurate, efficient and effective method to improve the quality of the myoelectric signal before use in prosthetic device for transhumeral amputees. Classification of the signal from upper limb using Surface Electromyogram (SEMG) signal has been the matter of extensive research. Discrete Wavelet Transform (DWT) coefficients along with the features values were extracted from the given SEMG data. With the appropriate choice of the mother wavelet, different shoulder motions were classified with the wavelet coefficients. In order to improve the class separability, analysis of variance method was used. It was found that the different motion can be identified accurately and provide the fundamental information to develop an efficient prosthetic device.
\end{abstract}

Keywords: Discrete Wavelet Transform, mother wavelet, Surface Electromyogram Signal, analysis of variance.

\section{Introduction}

Myoelectric control is an advanced technique typically used in the rehabilitation devices, exoskeletons, robots and a number of clinical applications. It seems to be the most used approach for amputees who have strong demand to improve their life. Existing muscles of amputees are used to control the prosthetic device. The initial step for controlling an active prosthetic device is to extract different features from the SEMG signal and then classify the signal for the different arm/hand motion.

In transhumeral amputees (Standard length is $50-90 \%$ of humeral length) the muscle activity of the phantom limb decreases, therefore the affected muscles cannot be perfectly used to control the prosthetic device operation. For these types of person, the arm/hand movement of the prosthesis can be controlled by around shoulder muscles, which is the main challenge in this study. The main difficulty in extracting the signal from the upper-limb amputee includes the electrode placement around the shoulder and the classification of the acquired SEMG signal's with respect to the motion of the shoulder [1]. Selection of shoulder muscles poses a great challenge in providing enough information for predicting the intended movement. Previous researchers have been focused on the hand motion classification of upper limb amputation by developing and optimizing of the SEMG pattern recognition system [2]. They classified the different hand motions from biceps brachii and triceps brachii and to classify the more complex hand motions with higher accuracy [3]. But the researchers did not pay attention to such person who has no activity of biceps and triceps

\footnotetext{
*E-mail address: laman.preet@thapar.edo

ISSN: $1791-2377$ C 2017 Eastern Macedonia and Thrace Institute of Technology. All rights reserved. doi:10.25103/iestr.104.15
}

muscles due to $60-90 \%$ above elbow arm amputation. Most of the daily life activities need hand-arm-shoulder complex structure for natural continuity of the body structure [4]. So the main aim of this study to analyze the movement of the shoulder with the activity of around shoulder muscles. These movements can help to control the hand motion activity for upper limb amputees.

To obtain more information from underlying muscles, number of signal processing techniques are applied to feature extraction. Various time domain analysis, frequency domain analysis and time-frequency domain representation have been compared during the feature extraction process $[1,5]$. From these analyses, time -frequency domain features show better results in case of muscle fatigue assessment. Wavelet Transform (WT) is one of the frequency time domain analysis methods. WT can be divided into discrete form and continues form. Discrete wavelet transform (DWT) has become an efficient tool to extract the features from the non stationary signal including the SEMG signal [6-10]. It provides adequate information for analysis of the original signal along with reduced computation time. DWT consists of lesser redundancy and comparatively easier to implement [7] [9].

In this present work different wavelet families were applied for SEMG signal classification. Root mean square value is the most significant parameter to quantify the most effective wavelet function. To determine the EMG signal's class separability for different operations of the arm, analysis of variance technique (ANOVA) was carried out from the experimental data. It compares the data of three groups and checks the effectiveness of the signal for three muscles Trapezius, Pectoral, and infraspinatus for different movements.

This paper is organized as follows. Section 2 describes the SEMG acquisition protocol detail. Section 3 and Section 4 briefly introduce the wavelet transform and statistical 
analysis respectively. Section 4 the detail description of experimental results.

\section{SEMG data Acquisition Protocol}

SEMG is generated during the muscles activation process are mainly collected by the non- invasiveness and convenient nature. The muscle activation in amputee and normal persons are totally different [1]. The SEMG signal used in this study was recorded from the five right upper limb amputee male people in the age group $35 \pm 5$ years and weight group $60 \pm 13 \mathrm{~kg}$. Data were accumulated using the NeXus-10 Mark II system. The demographic data of the amputees are shown in the Table 1.

Table 1. Demographic information about the participating amputee persons

\begin{tabular}{l|l|l|l}
\hline Person ID & Age (Years) & $\begin{array}{l}\text { Missing } \\
\text { Arm }\end{array}$ & $\begin{array}{l}\text { Time since } \\
\text { amputation }\end{array}$ \\
\hline A1 & 32 & Right & 9 years ago \\
\hline A2 & 38 & Right & 7 years ago \\
\hline A3 & 34 & Right & 6 years ago \\
\hline A4 & 40 & Right & 12 years \\
\hline A5 & 37 & Right & 10 years ago \\
\hline
\end{tabular}

Prior to the arrangement of $\mathrm{Ag} / \mathrm{AgCl}$ circular (oval) electrodes [1] on the body surface, oil or dust was taken away from the skin surface with an alcohol swab [13]. The standards for collection of SEMG signal are followed from the SENIAM project (Surface Electro-myography for the Noninvasive Assessment of Muscles) which is a European concerted action in the Biomedical Health and Research Program (BIOMED II) of the European Union [13]. The system was used to acquire the data from the three aroundshoulder muscles; infraspinatus muscle (I) (sensor 5,6), trapezius muscle $(\mathrm{T})$ (sensor 1,2), and pectoral muscle $(\mathrm{P})$ (sensor 3,4) from the right arm of the person as shown in Fig. 1. With three motions protraction, retraction and elevation of the shoulder. The detailed description of different shoulder movements with the involved muscles is shown in Table 2.

Table 2. Different shoulder movement and muscles involved

\begin{tabular}{l|l|l}
\hline $\begin{array}{l}\text { Shoulder } \\
\text { movements }\end{array}$ & Description & $\begin{array}{l}\text { Muscles } \\
\text { involved }\end{array}$ \\
\hline Resting & $\begin{array}{l}\text { The subject relaxed his } \\
\text { shoulder without any } \\
\text { activation of muscles }\end{array}$ & None \\
\hline $\begin{array}{l}\text { Elevation } \\
\text { EL) }\end{array}$ & $\begin{array}{l}\text { Lifted his shoulder towards } \\
\text { his ear softly and then } \\
\text { slowly loosened up to the } \\
\text { resting place. }\end{array}$ & Trapezius \\
\hline $\begin{array}{l}\text { Protraction } \\
\text { PT) }\end{array}$ & $\begin{array}{l}\text { Rolled them forwards, } \\
\text { squeezed the shoulder } \\
\text { blades at the chest }\end{array}$ & Pectoral \\
\hline $\begin{array}{l}\text { Retraction } \\
\text { RT) }\end{array}$ & $\begin{array}{l}\text { Rolled them in backwards, } \\
\text { squeezed the shoulder } \\
\text { blades at the back }\end{array}$ & Infraspinatus \\
\hline
\end{tabular}

All the subjects were instructed to perform exact shoulder movements in three different defined ways. Each movement was randomly repeated 4 times and recorded for 5 Sec. Each single movement generates a $[(m * n) * N]$ matrix where $m=$ the registration length, $n=$ sampling rate and $\mathrm{N}=$ no. of sensors. The SEMG system has gain factor of 19.5 and common mode rejection ratio was $\geq 100 \mathrm{~dB}$. The sampling rate of SEMG signal was kept 1024 samples/second for each channel and the resolution was 12.2 $\mathrm{nV} /$ bit. The raw SEMG signals were filtered by the IIR band pass Butterworth filter with frequency range 20-500 Hz.

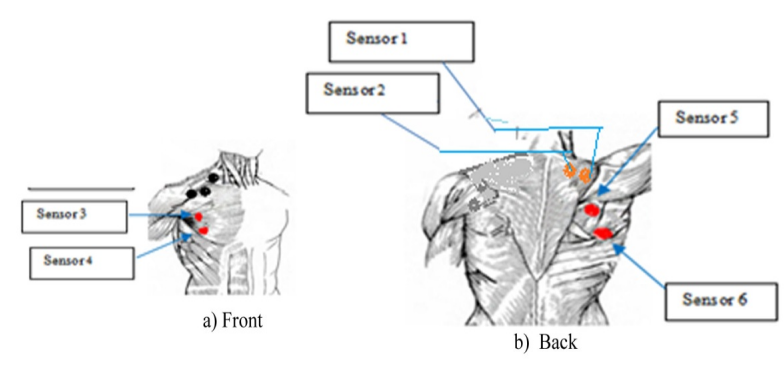

Fig. 1. Location of sensors around the shoulder

\section{DWT based Feature Extraction:}

DWT is a multi-resolution technique used in real time engineering applications. It exhibits good time resolution for high frequency signal and good frequency resolution at low frequency [12]. Thus, the low frequency components are more significant than high frequency elements. Thus, DWT is characterized by the inner product of dilatation of the mother wavelet $\psi$ that is directly deduced from scaling function $\varphi$ and the basis function. The wavelet $\psi_{\mathrm{x}, \mathrm{y}}(\mathrm{n})$ is derived from the mother wavelet $\psi($.$) .$

The relation can be defined as:

$\psi x, y(t)=\left(\frac{1}{\sqrt{x}}\right) \times \psi\left(\frac{\mathrm{n}-\mathrm{y}}{\mathrm{x}}\right)$

Where $\mathrm{x}$ and $\mathrm{y}$ is the scaling factor and translation factor respectively. The function generated by can be :

$\cap=\{\psi x, y(t), x \quad R, y=R, a \neq 0\}$

$\cap$ is called frame of $D^{2}(R)$ and it should be satisfy the following condition

$$
\psi=\int_{0}^{\infty} \frac{\psi(\omega)^{2}}{\omega} \mathrm{d} \omega<\infty
$$

Where, $\psi(\omega)$ is generated from fourier transform of $\psi(t)$. The function $\mathrm{f}(\mathrm{t}) \varepsilon \mathrm{D}^{2}(\mathrm{R})$ then its countinuous wavelet transform is the inner product of $\mathrm{f}(\mathrm{t})$ and $\psi_{\mathrm{x}, \mathrm{y}}(\mathrm{t})$.

Therefore the DWT equation of a signal $f(n)$ is defined as :

$\operatorname{DWT}(i, j)=\left(\frac{1}{\sqrt{x}}\right) \sum_{r} \psi\left(\frac{r-b}{x}\right) f(n) \quad a>0$

A wavelet approach exhibit superior performance and well suited to non-stationary signal. The original signal is decomposed into various detail and approximate signal level. The detail parts consist of high frequency signal and approximate part consist of low frequency signal.

For the feature vector selection, the maximum absolute value of the approximate coefficient with last level and marked as LLL. SEMG signal was decomposed by DWT 3 level. Different wavelet function affects the performance of 
WT. So it was very important to choose the best and appropriate wavelet function. After the features vector results, we need to determine the type of mother wavelet to maintain the accurate classification accuracy. For best wavelet, the different wavelet functions were compared and select the wavelet function with maximum root mean square value.

\section{Statistical Analysis.}

ANOVA is a statistical technique for evaluating the significance of the class separability by taking the means $(\mu)$ of several groups of data. The data are classified according to the two factors, therefore two way ANOVA with replication was utilized for separability of classes.

Assume that for $\mathrm{n}$ observations where row ( $\mathrm{r}$ ) has $1,2,3, \ldots \ldots$. A level and column (c) has $1,2,3 \ldots .$. . level sample number with $\mathrm{p}$ values of independent observation (called replication). The null $\left(\mathrm{H}_{0}\right)$ and alternate hypotheses $\left(\mathrm{H}_{1}\right)$ of interest in an ANOVA are as follows:

$\mathrm{H}_{01}: \mu_{1}=\mu_{2}=\mu_{3} \ldots=\mu_{\mathrm{a}} \mathrm{H}_{\mathrm{A} 1} \quad:$ all $\mu_{\mathrm{i}}^{\prime}$ s are not equal.

$\mathrm{H}_{02}: \mu_{1}=\mu_{2}=\mu_{3} \ldots=\mu_{\mathrm{b}} \mathrm{H}_{\mathrm{B} 1} \quad$ : all $\mu_{\mathrm{i}}$ 's are not equal.

The technique involves the following steps :

(i) Calculate total of all observations for each of 'rp' A class

(ii) Calculate total of all observations for each of the 'cp' B class

(iii) Calculate the total of all observations for each level called cell sums of the AB class

(iv) Calculate Grand Total (G)

$\mathrm{T}_{00}=\sum_{i} \sum_{j} Y i j$

(iv) Calculate Correction factor (C.F)

$\mathrm{G}^{2} / \mathrm{N}$ where $\mathrm{N}=\mathrm{rcp}$

(v) Total sum of squares $\left(\mathrm{TSS}=\sum_{i} \sum_{j} Y i j^{2}-\mathrm{C} . \mathrm{F}\right.$

(vi) Sum of squares due to rows (SSR)

$=\frac{\sum_{\mathrm{i}} \mathrm{Tio}^{2}}{\mathrm{cp}}-\mathrm{C} . \mathrm{F}$

(vii) Sum of squares due to columns (SSC) $=\frac{\sum_{j} T o j^{2}}{r p}-$ C.F

(viii) Sum due to the combined rows and columns $(\mathrm{SSRC})=\frac{\sum_{i} T o i^{2} \sum_{j} T o j^{2}}{p}-\mathrm{C} . \mathrm{F}-\mathrm{SSR}-\mathrm{SSC}$

(viii) Sum of squares due to error $(\mathrm{SSE})=\mathrm{TSS}-\mathrm{SSR}-\mathrm{SSC}$ - SSRC

(ix) Prepare ANOVA Table as shown in Table 1.

The calculated values are compared with the tabulated values. If the calculated value of $\mathrm{F}$ is greater than the critical value, then the hypothesis $\mathrm{H}_{\mathrm{o} 1}$ and $\mathrm{H}_{02}$ will be rejected otherwise accepted. The significance level $\alpha=0.05$, is a statistical evidence level, which shows the difference in mean values. In statistical hypothesis testing, a p-value (probability value) is a numerical measure of the statistical significance which helps to provide the strongest evidence against the null hypothesis of the data samples.

Table 3. Two Way ANOVA Computational Table

\begin{tabular}{|c|c|c|c|c|c|}
\hline $\begin{array}{l}\text { Source of } \\
\text { variation }\end{array}$ & $\begin{array}{l}\text { Degree } \\
\text { of } \\
\text { freedom } \\
\text { (df) } \\
\end{array}$ & $\begin{array}{l}\text { Sum of } \\
\text { squares } \\
\text { (SS) }\end{array}$ & $\begin{array}{l}\text { Mean } \\
\text { sum of } \\
\text { squares } \\
\text { (MSS) }\end{array}$ & F ratio & $\begin{array}{l}P \\
\text { value }\end{array}$ \\
\hline $\begin{array}{l}\text { Level } \\
\text { A(rows) }\end{array}$ & $r-1$ & SSR & MSSR & MSSR /MSSE & Tail \\
\hline Level & $\mathrm{c}-1$ & $\mathrm{SSC}$ & MSSC & MSSC/MSSE & Tail \\
\hline Interaction & $\begin{array}{l}(\mathrm{r}-1)(\mathrm{c}- \\
1)\end{array}$ & SSRC & & MSSRC/MSSE & $\begin{array}{l}\text { Tail } \\
\text { area }\end{array}$ \\
\hline $\begin{array}{l}\text { With in } \\
\text { (Error) } \\
\text { Total }\end{array}$ & $\begin{array}{l}\mathrm{rc}(\mathrm{n}-1) \\
\mathrm{rcn}-1\end{array}$ & $\begin{array}{l}\text { SSE } \\
\text { TSS }\end{array}$ & MSSE & & \\
\hline
\end{tabular}

In this study, if the $\mathrm{p}$-value is less than $5 \%(\mathrm{p}<0.05)$, then the null hypothesis is assumed to be rejected or the results are statistically significant.

\section{Experimental Results and Discussions}

\subsection{Wavelet Analysis}

To classify the shoulder movement, there are four steps: 1) acquiring the SEMG signal b) extracting feature vector using best mother wavelet c) separability with statistical techniques.

The time-frequency-based analysis were used to extract the SEMG features. For the interpretation of the signal, the amplitude variation estimation is more significant for identifying the prominent location of the muscles. Root mean square (RMS) measures the magnitude of the variable quantity.

Table 4. Signal features on different muscles for different motions of shoulder

\begin{tabular}{l|l|l|l|l}
\hline $\begin{array}{l}\text { Motions of } \\
\text { shoulder }\end{array}$ & $\begin{array}{l}\text { SEMG } \\
\text { signal level }\end{array}$ & $\begin{array}{l}\text { RMS } \\
\text { (Trapezious) }\end{array}$ & $\begin{array}{l}\text { RMS } \\
\text { (Pectorial) }\end{array}$ & $\begin{array}{l}\text { RMS } \\
\text { (Infraspinatus) }\end{array}$ \\
\hline Elevation & High & 0.0347 & 0.015 & 0.0104 \\
Protraction & Low & 0.0152 & 0.019 & 0.0059 \\
Retraction & Medium & 0.0233 & 0.0089 & 0.0263 \\
\hline
\end{tabular}

A large superficial Trapezius muscle plays a crucial role in maintaining the proper shoulder motion and have a higher muscle activation during elevation motion means high level SEMG signal. The activity of the infraspinatus muscle is less than trapezious but more than pectorial muscle. However, the deviation between the different RMS values (corresponding to muscle activation) is very small as shown in table 4 , so it is difficult to utilize this data for controlling the prosthetic device.

During the extraction process of SEMG signal, DWT with four level wavelet function was used to extract the features in the time - frequency domain. The feature vector of LLL was used. The DWT level 4 [11] decomposed SEMG signal detected from trapezius muscle illustrated in figure 2 . 


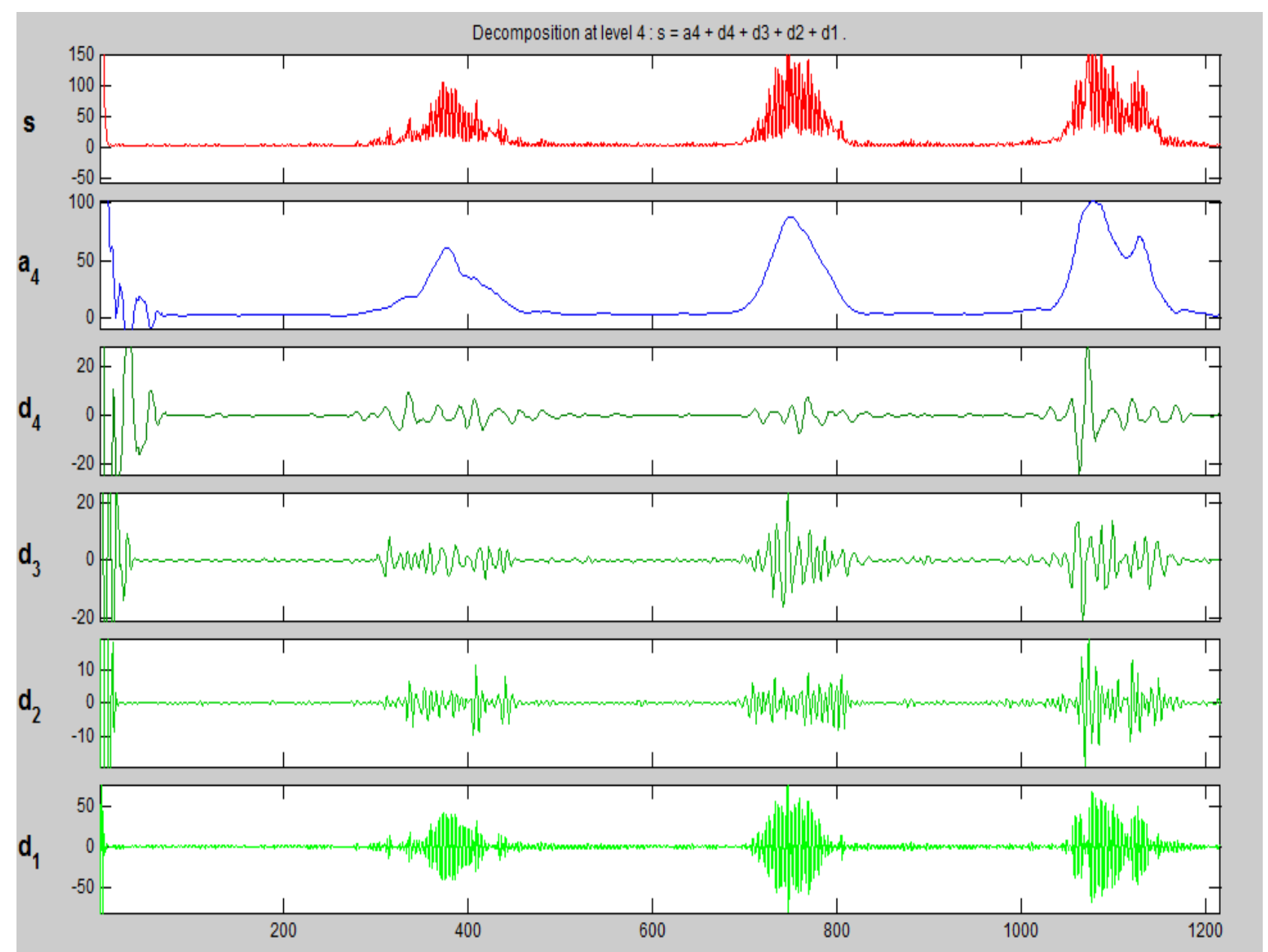

Fig. 2. SEMG signal usings wavelet analysis with $\mathrm{dB} 4$ wavelet with 4 level decomposition from EL. In which $\mathrm{d} 1, \mathrm{~d} 2, \mathrm{~d} 3$, d4 is the details at the first, second, third and fourth level respectively. a4 is the approximation at third level. $\mathrm{s}$ is the original signal.

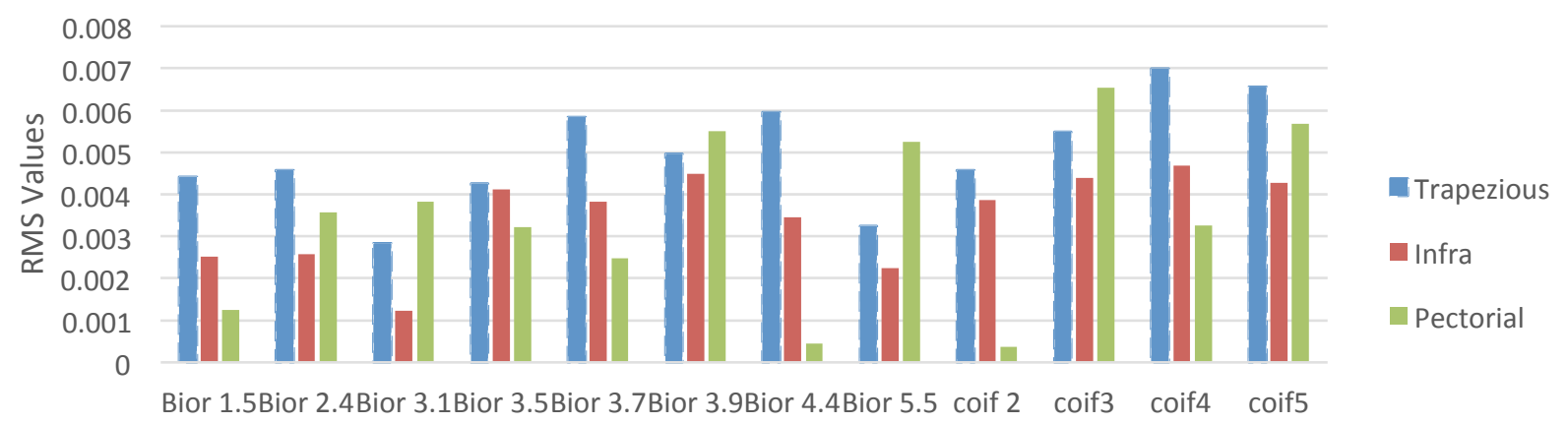

Mother Wavelet

Fig. 3. RMS value of mother wavelet selection. Bior belongs to biorthogonal wavelets and coif belongs to coiflet wavelets.

On the basis of feature vector LLL the two wavelet family with different order are determined with their RMS value Figure 3 depicts the average RMS value of the five subjects with the biorthognal (bior) wavelet functions and coiflet (coif) wavelet for three defined muscles. From these values the coif 4 shows better performance than bior family, is selected as the best mother wavelet.

For better visualization, wavelet coefficients of selected mother wavelet were considered for classification. The figure 4 shows the coif 4 wavelet coefficient values of the SEMG data set at different muscles with different motion. Trapezius muscles activation is maximum with the elevation motion of arm. The overall activation of infraspinatus muscle was less as compared to other muscles but gave maximum value with retraction motion. Figure 4 presented the actual classified muscles value with different motion of shoulder.

The proposed coefficient values with selected mother wavelet can efficiently separate the activation of the three muscle groups.

\subsection{Statistical Variance Analysis Technique}

To examine the amount of variance in each sample to establish more confidence into actions of different muscles statistics based variance analysis technique (ANOVA) was carried out for the analysis of a signal's class separability from the observational data. ANOVA determines whether the remainder between the samples is due to an error or has some significant value. The row vectors are the different 
muscles, and the column vectors are the different movements of the shoulder that provide the $3 * 3$ ANOVA matrix.

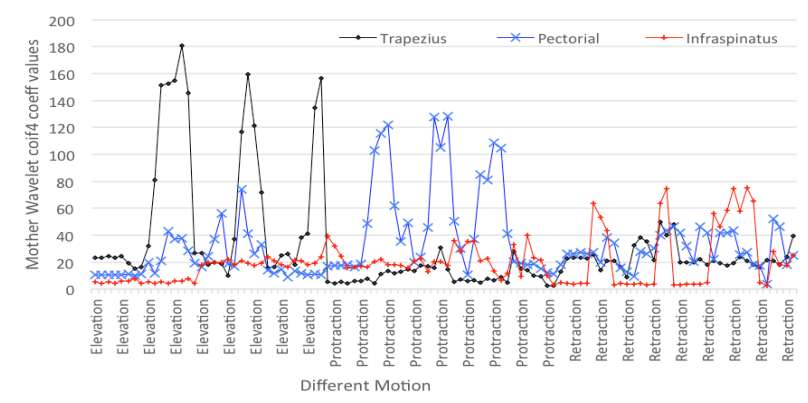

Fig. 4. Trapezius, Pectoral and Infraspinatus muscle activation using wavelet coefficient values
Each group data variation is estimated for the three individual muscles. A number of tests are considered to find out which test samples are significant at this level. The mean square value within the groups for the Trapezius, pectorals, and infraspinatus is shown in Table 4. The tabulated value or critical value and calculated values for both columns and rows at 5\% significance level.

Table 5. ANOVA: Two-factor with replication

\begin{tabular}{|c|c|c|c|c|}
\hline SUMMARY & Elevation & Protraction & Retraction & Total \\
\hline \multicolumn{5}{|l|}{ Trapezius } \\
\hline Count & 12 & 12 & 12 & 36 \\
\hline Sum & 0.849 & 0.164 & 0.484 & 1.497 \\
\hline Average & 0.0707 & 0.0137 & 0.040 & 0.042 \\
\hline Variance & $304 \mathrm{E}-05$ & 7.44E-05 & 2.19E-05 & $685 \mathrm{E}-05$ \\
\hline \multicolumn{5}{|l|}{ Infraspinatus } \\
\hline Count & 12 & 12 & 12 & 36 \\
\hline Sum & 0.828 & 0.045 & 1.0274 & 1.900 \\
\hline Average & 0.069 & 0.0037 & 0.086 & 0.053 \\
\hline Variance & 591E-06 & 3.3E-06 & $145 \mathrm{E}-06$ & $152 \mathrm{E}-05$ \\
\hline \multicolumn{5}{|l|}{ Pectoralis } \\
\hline Count & 12 & 12 & 12 & 36 \\
\hline Sum & 0.198 & 0.489 & 0.342 & 1.02871 \\
\hline Average & 0.0165 & 0.041 & 0.028 & 0.028575 \\
\hline Variance & $1.227 \mathrm{E}-05$ & $6.85 \mathrm{E}-05$ & $1.71 \mathrm{E}-05$ & $132 \mathrm{E}-06$ \\
\hline \multicolumn{5}{|l|}{ Total } \\
\hline Count & 36 & 36 & 36 & \\
\hline Sum & 1.88 & 0.699 & 1.852664 & \\
\hline Average & 0.0521 & 0.019 & 0.051463 & \\
\hline Variance & $94 \mathrm{E}-05$ & 299E-05 & 69E-05 & \\
\hline
\end{tabular}

\begin{tabular}{|c|c|c|c|c|c|}
\hline $\begin{array}{l}\text { Source of } \\
\text { Variation }\end{array}$ & $\begin{array}{l}\text { Sum of } \\
\text { squares }\end{array}$ & Degree of freedom & Mean sum of squares & F Ratio & $\mathrm{P}$ value \\
\hline Sample & 0.01066 & 2 & 0.0053 & 38.4 & $4.52 \mathrm{E}-13$ \\
\hline Columns & 0.0262 & 2 & 0.0126 & 91.5 & $3.17 \mathrm{E}-23$ \\
\hline Interaction & 0.0429 & 4 & 0.011 & 78.0 & $9.66 \mathrm{E}-30$ \\
\hline Within & 0.01362 & 9999 & 0.00014 & & \\
\hline Total & 0.093 & 107 & & & \\
\hline
\end{tabular}

In ANOVA with replication, it was observed that the $\mathrm{F}(2,99)=38.4,91.5$ and $\mathrm{F}(4,99)=78.0$ and this $F$ ratio is greater than critical value 3.088 and 2.46 respectively as shown in Table 5 , so the means are significantly different.. The $\mathrm{p}$ value is less than $0.05 \%$ of significance level. So the null hypothesis was rejected and it was concluded that the three muscles gave the independent voluntary contraction with different movement and test statistics was different at this level.

\section{Conclusion}

This paper presented the influence of the SEMG signal on different muscles to estimate the effect of signal variation corresponding to the different motions of the upper limb.
The SEMG features were extracted from the multichannel SEMG setup successfully. DWT-based feature extraction schemes were proposed that provide the essential information. The selected mother wavelet accurately give the classification of muscle activity with the motion of the shoulder. Secondly, the results give the separability in motion using the analysis of variance technique. These strategies help to realize the activation of the different muscles for shoulder movement. Based on the above advantages, this method will be used to design myoelectric prosthetic arm for above elbow amputees.

This is an Open Access article distributed under the terms of the Creative Commons Attribution Licence

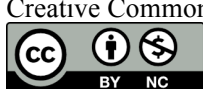




\section{References}

1. Kaur A., Agarwal R., Kumar A. (2016). Comparison of muscle activity of able bodied and amputee subjects for around shoulder movement. Biomed Mater Eng. 27(1), 29-37.

2. R Hardeep, Agarwal R., Arora A.S. (2010) Interpretation of wrist operations from SEMG signals at different locations on arm. IEEE Trans. Biomedical Circuits and Systems, 4 ,101-11.

3. Veer K., Agarwal R., Kumar, A. (2016). Processing and Interpretation of Surface Electromyogram Signal to Design Prosthetic Device. Robotics, 32 (07), 1486 - 1494.

4. Soma Hirokazu, HoriuchiYuse, Gonzalez Jose and Wenwei Yu. (2011) Classification of Upper Limb Motions from AroundShoulder Muscle Activities. Proceeding of IEEE Int. Conf. on Rehabilitation Robotics Rehab Week Zurich. ETH Zurich Science City, Switzerland. 310-15.

5. Kaur A., Agarwal R, Kumar, A. (2014). A Combined Statistical and Time-Frequency Approach to the Analysis of Electromyography Signals national conference on Advances in Metrology AdMet, Feb. 19-21,

6.A. Phinyomark1, C. Limsakul, P. (2011)PhukpattaranontApplication of Wavelet Analysis in EMG Feature Extraction for Pattern Classification, Measurement Science Review, volume 11, No. 2, 45-52.

7.Phinyomark, A., Hirunviriya, S., Limsakul, C., Phukpattaranont, P. (2010). Evaluation of EMG feature extraction for hand movement recognition based on euclidean distance and standard deviation. In 7th International Conference on Electrical Engineering/Electronics, Computer, Telecommunications and Information Technology, May 2010. Chiang Mai, Thailand, 856-860.

8 Wang, G., Wang, Z., Chen, W., Zhuang, J. (2006). Classification of surface EMG signals using optimal wavelet packet method based on Davies-Bouldin criterion. Med. Biol. Eng. Comput., 44 (4), 865 872.

9. A. Phinyomark, A. Nuidod, P. Phukpattaranont, C. Limsakul. Feature Extraction and Reduction of Wavelet Transform Coefficients for EMG Pattern Classification Electronics and electrical engg. 27-33

10. A. B. M. S. U., S. A. Fattah and M. O. Ahmad,Doulah. Domain Feature Extraction Scheme Basedon Dominant Motor Unit Action Potential of EMGSignal for Neuromuscular Disease Classification.IEEE Transactions On Biomedical Circuits And Systems, Vol. 8, No. 2, April 2014 11. Misiti, M., Misiti, Y., Oppenheim, G., Poggi, J.-M. (2010). Wavelet Toolbox User's Guide. The MathWorks, Inc. Retrieved June 28, 2010, from MathWorks http://www.mathworks.com/access/help desk/help/pdf_doc/wavelet/wavelet_ug.pdf.

12. Englehart, K., Hudgins, B., Parker, P.A. (2001). A wavelet-based continuous classification scheme for multifunction myoelectric control. IEEE Trans. Biomed. Eng., 48 (3), 302-311.

13.Merletti, R., 1999. Standards for Reporting EMG Data. Journal of Electromyography and Kinesiology, February 1999; 9(1):III-IV $<$ http://www.isek-online.org/standards_emg.html $>$ 